\title{
DNA methylation of CRB3 is a prognostic biomarker in clear cell renal cell carcinoma
}

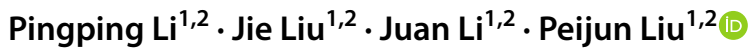

Received: 18 December 2018 / Accepted: 17 May 2019 / Published online: 30 May 2019

(c) The Author(s) 2019

\begin{abstract}
Our previous study revealed that CRB3 protein expression was reduced in clear cell renal cell carcinoma (ccRCC) and was associated with TNM stage, pathological grade, and poor prognosis of ccRCC. This study aimed to investigate if DNA methylation of CRB3 decreases its expression, subsequently leading to the progression and poor prognosis of ccRCC. Data for DNA methylation of CRB3, CRB3 mRNA expression, and ccRCC clinicopathological parameters were extracted from the cancer genome atlas (TCGA) database. The relationships among DNA methylation of CRB3, CRB3 mRNA expression, and ccRCC clinicopathological parameters were analyzed using UALCAN, MethHC, LinkedOmics, and Wanderer. We found that CRB3 mRNA levels were lower in ccRCC compared to normal tissues. Methylation of CRB3 increased in ccRCC, with all probes showing differences between ccRCC and normal tissues. Furthermore, CRB3 DNA methylation negatively correlated with CRB3 mRNA expression. CRB3 DNA methylation was also related to pathologic stage, $\mathrm{T}$ stage, $\mathrm{N}$ stage, and $\mathrm{M}$ stage of ccRCC. Overall survival was shorter in ccRCC patients with high CRB3 DNA methylation compared to ccRCC patients with low CRB3 DNA methylation. Methylation of cg24798010, a CRB3 probe, was related to laterality, pathologic stage, $\mathrm{T}$ stage, $\mathrm{M}$ stage, neoplasm-histologic-grade without $\mathrm{N}$ stage, and race. Furthermore, treatment with the DNA methylation inhibitor Decitabine resulted in the upregulation of CRB3 mRNA in ccRCC cell lines. These results indicate that DNA methylation of CRB3 may be both a prognostic marker and therapeutic target for ccRCC.
\end{abstract}

Keywords DNA methylation $\cdot$ Cell polarity protein CRB3 $\cdot$ Clear cell renal cell carcinoma $\cdot$ Prognosis

\section{Introduction}

Renal tumors are one of the ten most common cancers, accounting for $3.8 \%$ of all new cancer cases. In 2018, 2.5\% of all cancer-related mortality in the United States was attributed to renal tumors [1]. Clear cell renal cell carcinoma (ccRCC) is the most common subtype of renal cell carcinoma (RCC), accounting for $90 \%$ of all renal tumors [2].

Electronic supplementary material The online version of this article (https://doi.org/10.1007/s11033-019-04892-7) contains supplementary material, which is available to authorized users.

Peijun Liu

liupeijun@xjtu.edu.cn

1 Center for Translational Medicine, The First Affiliated Hospital of Xi' an Jiaotong University, 277 West Yanta Road, Xi' an 710061, Shaanxi, China

2 Key Laboratory for Tumor Precision Medicine of Shaanxi Province, The First Affiliated Hospital of Xi' an Jiaotong University, Xi'an, Shaanxi, China
About $65 \%$ of RCC are localized tumors, which can be effectively treated with surgery. However, $30 \%$ of non-metastatic ccRCC recur after (partial) nephrectomy. In contrast, 35\% of RCC are metastatic RCC (mRCC), and 5-year survival of metastatic ccRCC is only $12 \%$ [3]. RCC is not sensitive to chemoradiotherapy, so therapeutic agents (kinase and immune checkpoint inhibitors) are the first-line treatment for patients with $\mathrm{mRCC}$ and for patients who relapse after surgery [4-7]. However, some therapeutic agents are associated with varying toxicities, drug resistance, and financial burden [2]. Thus, there is a clinical urgency to identify tumor markers for preliminary screening, early detection of metastasis, and guidelines for drug development and clinical use for ccRCC.

Epigenetic modification refers to heritable changes in gene expression levels that do not alter the DNA sequence [8]. DNA methylation is an epigenetic modification that has been extensively studied [9], and which can contribute to renal tumorigenesis by silencing tumor suppressor genes 
$[10,11]$. Furthermore, DNA methylation is associated with clinicopathological features and poor prognosis $[11,12]$.

Cell polarity protein CRB3, a member of the Crumbs family, is found in mammalian epithelia [13]. CRB3 determines epithelial cell polarity at the apical surface and plays a role in the formation of tight junctions [14, 15]. CRB3 is also highly expressed in the kidneys, where it establishes and maintains the polarity of kidney embryos [16]. Overexpression of CRB3 in renal epithelial Hela cells improves cell to cell cohesion and changes the cytoskeleton [17]. Furthermore, knockout of CRB3 causes polycystic kidney disease in mice $[18,19]$.

CRB3 acts as a tumor suppressor in renal tumors [20, 21]. CRB3 protein expression was reduced in ccRCC tissues compared to adjacent normal kidney tissue samples, and was negatively associated with TNM stage, pathological grade, and poor prognosis of ccRCC patients [21]. Repression of CRB3 mRNA and protein led to immortality of mouse kidney epithelial cells; rescued CRB3 expression restored cell junctions, cell polarity, and contact inhibition, and suppressed migration and metastasis of tumor cells [20].

DNA methylation is stable compared to gene expression [22] and is a key DNA modification that stabilizes gene silencing and defines cell fate [23]. Given that DNA methylation has been suggested to silence tumor suppressor genes [10,11], we postulated that CRB3 DNA methylation down-regulates $\mathrm{CRB} 3$ protein expression and serves as a prognostic marker for ccRCC. In this study, the relationship among DNA methylation of CRB3, CRB3 expression levels, and clinical pathological parameters in ccRCC were investigated utilizing TCGA datasets.

\section{Materials and methods}

\section{mRNA expression of CRB3}

UALCAN (http://ualcan.path.uab.edu/index.html) is a web tool that can be used to analyze tumor transcriptome data. The web tool provides: (1) publicly accessible cancer transcriptome data (e.g.: TCGA mRNA sequencing), and (2) published graphs and plots depicting gene expression and patient survival information [24]. mRNA expression of CRB3 in normal and tumor specimens of ccRCC was comparatively analyzed using the UALCAN web tool.

\section{CRB3 methylation analysis}

Wanderer (http://maplab.imppc.org/wanderer/) is a web tool that can be used to analyze gene expression and DNA methylation profiles from TCGA. The web tool provides the DNA methylation levels of Illumina Human Methylation 450 Bead Chip loci within or close to the queried gene [25].
Differences in CRB3 methylation were obtained through analysis of the TCGA ccRCC cancer (KIRC, Kidney renal clear cell carcinoma) dataset. Correlations between methylation and CRB3 gene expression were tested using the Spearman (r) correlation method. Correlations were also examined between individual probes with methylation changes and mRNA expression. A correlation was considered either strong ( $\mathrm{r}=0.5$ to 1.0 or -0.5 to -1.0$)$, moderate, $(r=0.3$ to 0.5 or -0.3 to -0.5 ), or weak ( $r=0.1$ to 0.3 or -0.1 to $-0.3)$ [26].

MethHC (http://methhc.mbc.nctu.edu.tw/php/index.php) is a web-based resource that focuses on DNA methylation. MethHC integrates data including gene expression, DNA methylation, microRNA expression, microRNA methylation, and the correlation of methylation and gene expression from TCGA.

\section{Association of CRB3 DNA methylation with pathological features and overall survival in CCRCC}

We used the web tool, LinkedOmics (http://www.linkedomic s.org/login.php) to analyze the multi-omics data from all 32 TCGA cancer types. Using LinkedOmics three analytical modules, we identified and analyzed information about mRNA or protein expression signatures, biomarkers of clinical attributes, and putative target genes of transcriptional factors, microRNAs, or protein kinases; analysis results can be depicted by plots [27]. The association of CRB3 DNA methylation with pathological features and overall survival in ccRCC was analyzed using the LinkedOmics web tool with Methylation450 and clinical data with non-parametric analysis.

\section{Cell culture}

Human clear cell renal cell carcinoma (ccRCC) cell lines Caki-1 and Caki-2 were obtained from the National Infrastructure of Cell Line Resource (Beijing, China). Caki-1 and Caki-2 cells were cultured in McCoy'5A Media (Hyclone) supplemented with $10 \%$ FBS (Hyclone) in a humidified atmosphere containing $5 \% \mathrm{CO}_{2}$ at $37{ }^{\circ} \mathrm{C}$.

\section{DNA methylation inhibitor treatment}

The same numbers of cells were seeded in each well of 6-well plates and cultured at $37^{\circ} \mathrm{C}$ in the medium containing 1,5 , or $10 \mu \mathrm{M}$ Decitabine (Selleck, Houston, TX, USA) or vehicle (DMSO). The medium was refreshed every $24 \mathrm{~h}$ during a 72-h period. Cells were then harvested for quantification of the mRNA level of CRB3. 


\section{Quantitative real time RT- PCR (RT-qPCR)}

Total RNA was isolated from cells using the RNA Fast 200 (\#220010, Fastagen Biotech, Shanghai, China) and reversely transcribed into cDNA using the PrimeScript ${ }^{\mathrm{TM}}$ RT Master Mix (\#RR036A, TaKaRa Biotechnology (Dalian) Co., Ltd. Liaoning, China) according to the manufacturers' protocols. qPCR was performed using the TB Green ${ }^{\mathrm{TM}}$ Premix Ex Taq ${ }^{\mathrm{TM}}$ II (\#RR820A, TaKaRa) in Bio-Rad CFX96 system (Hercules, CA) according to the manufacturer's instructions. The primers used were: CRB3, 5'-CTT CTG CAA ATG AGA ATA GCA CTG-3' and 5'-GAA GAC CAC GAT GAT AGC AGT GA-3'; $\beta$-actin, 5 '-CAT GTA CGT TGC TAT CCA GGC-3' and 5'-CTC CTT AAT GTC ACG CAC GAT-3'. The experiment was performed in triplicate and level of CRB mRNA was normalized to $\beta$-actin using the $2^{-\Delta \mathrm{Ct}}$ method.

\section{Statistical analysis}

GraphPad Prism Version 7.00 was used for statistical analyses. Statistically significant differences between two groups were tested by the Mann-Whitney test; significant differences among three groups were compared using one-way analysis of variance (ANOVA) followed by Dunnett's multiple comparisons test; four groups were determined by the Kruskal-Wallis test. Correlation of the overall survival with CRB3 DNA methylation was tested using the Cox Regression test.

\section{Results}

\section{CRB3 mRNA expression in ccRCC}

CRB3 mRNA expression was analyzed in normal and ccRCC tumor specimens using the UALCAN web tool. We found that CRB3 mRNA levels were significantly $(P<0.05)$ reduced in the ccRCC tissues compared to the normal tissues (normal $n=72$, tumor $n=533$ ) (Fig. 1).

\section{CRB3 methylation in ccRCC}

We analyzed CRB3 methylation in normal and ccRCC tumor specimens using the Wanderer web tool. We found that CRB3 methylation was significantly $(P<0.05)$ increased in ccRCC (normal $n=160$, tumor $n=324$ ), and all of the CRB3 probes in the 450 Methylation array showed significant differences between ccRCC and normal specimens (Fig. 2). DNA methylation of the CRB3 probes is shown in Table 1.

The correlation between DNA methylation and CRB3 mRNA expression in ccRCC was analyzed using the MethHC web tool. The specific $P$ values are shown in
Expression of CRB3 in KIRC based on major cancer stages

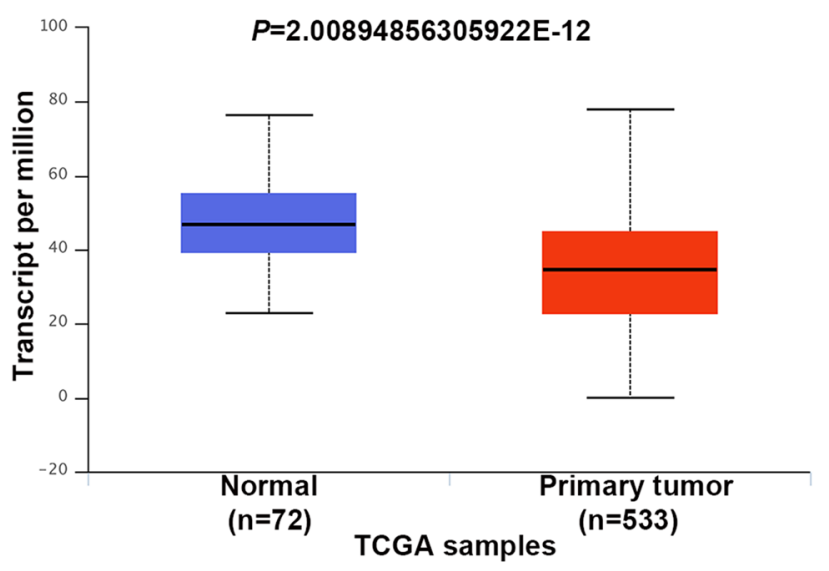

Fig. 1 CRB3 mRNA expression in ccRCC (kidney renal cell carcinoma, KIRC). Box plot and $P$ value were produced using UALCAN (http://ualcan.path.uab.edu/index.html). $P=2.00894856305922 \mathrm{e}-12$

Table 2. CRB3 DNA methylation was negatively correlated with CRB3 mRNA expression (Table 3).

\section{Association of CRB3 DNA methylation and pathological features in CCRCC}

We analyzed the association of CRB3 DNA methylation (all probes) and pathological features in ccRCC using the LinkedOmics web tool (Fig. 3). CRB3 DNA methylation (all probes) correlated with pathologic stage $(P=2.134 \mathrm{e}-04$, $\mathrm{n}=318), \mathrm{T}$ stage $(P=1.004 \mathrm{e}-04, \mathrm{n}=319), \mathrm{N}$ stage $(P=2.383 \mathrm{e}-02, \mathrm{n}=141)$, and $\mathrm{M}$ stage $(P=1.488 \mathrm{e}-02$, $\mathrm{n}=287$ ) (Fig. 3). These results indicate that DNA methylation of CRB3 correlates with ccRCC progression.

\section{Association of CRB3 DNA methylation and ccRCC prognosis}

We analyzed the association of CRB3 DNA methylation and ccRCC prognosis using the LinkedOmics web tool (Fig. 4). CRB3 DNA methylation significantly $(P<0.05)$ affected the overall survival of ccRCC patients. Overall survival was significantly shorter for ccRCC patients with high CRB3 DNA methylation compared to ccRCC patients with low CRB3 DNA methylation (Logrank $P=5.799 \mathrm{e}-07, \mathrm{n}=307$ ). These results indicate that CRB3 DNA methylation related with poor ccRCC prognosis.

\section{Correlation between individual probe methylation and CRB3 mRNA}

All of the CRB3 probes in the 450 Methylation array were further analyzed for their correlations with CRB3 mRNA expression (Table 3). There was a moderate or weak 


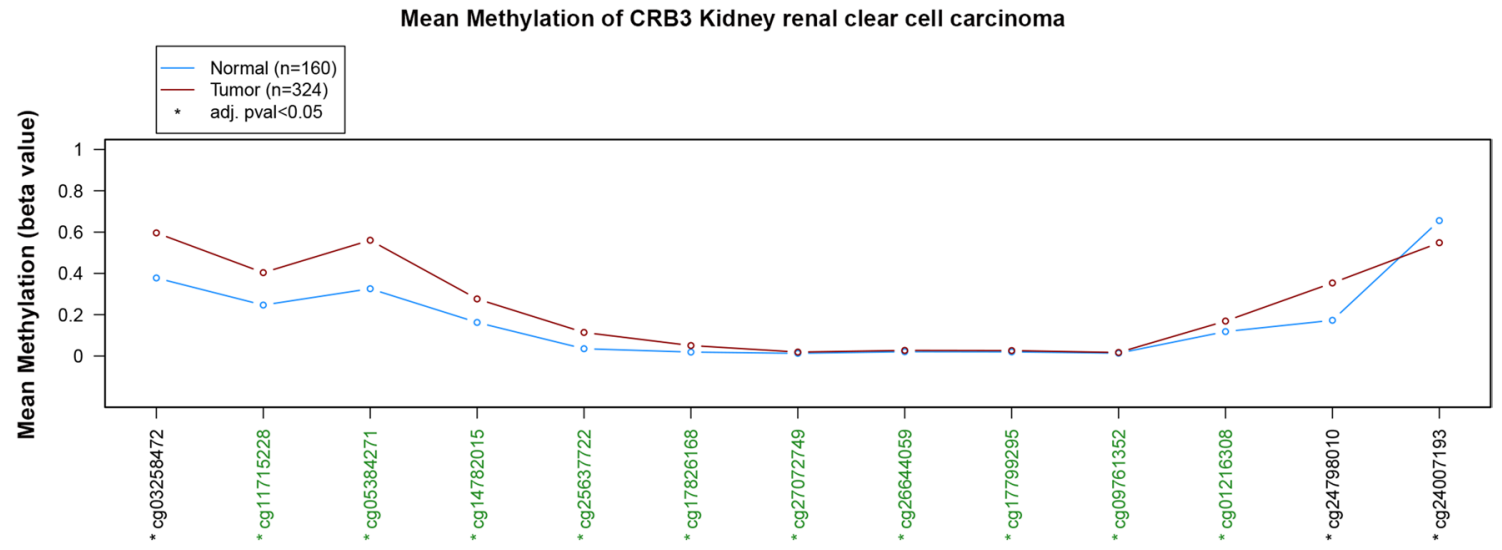

Fig. 2 Mean CRB3 DNA methylation in ccRCC. Green font represents $\mathrm{CpG}$ islands; the adj.pval represents adjusted $P$-value and $*$ represents adjusted $P$-value $<0.05$. Plot and $P$ values were produced using Wanderer (http://maplab.imppc.org/wanderer/)

Table 1 CRB3 DNA methylation in normal tissues $v s$ ccRCC tumor specimens

\begin{tabular}{llll}
\hline Probe & Gene name & Wilcox_stat & Adj.pval \\
\hline $\operatorname{cg} 03258472$ & CRB3 & 4653.5 & $9.47 \mathrm{E}-48$ \\
$\operatorname{cg} 11715228$ & CRB3 & 9663.5 & $4.17 \mathrm{E}-29$ \\
$\operatorname{cg} 05384271$ & CRB3 & 5234.5 & $1.63 \mathrm{E}-45$ \\
$\operatorname{cg} 14782015$ & CRB3 & 5469 & $1.10 \mathrm{E}-44$ \\
$\operatorname{cg} 25637722$ & CRB3 & 6051 & $2.30 \mathrm{E}-42$ \\
$\operatorname{cg} 17826168$ & CRB3 & 6831 & $2.70 \mathrm{E}-39$ \\
$\operatorname{cg} 27072749$ & CRB3 & 11007 & $8.94 \mathrm{E}-25$ \\
$\operatorname{cg} 26644059$ & CRB3 & 14770.5 & $1.34 \mathrm{E}-14$ \\
$\operatorname{cg} 17799295$ & CRB3 & 12853.5 & $2.09 \mathrm{E}-19$ \\
$\operatorname{cg} 09761352$ & CRB3 & 13378 & $4.92 \mathrm{E}-18$ \\
$\operatorname{cg} 01216308$ & CRB3 & 7806 & $1.17 \mathrm{E}-35$ \\
$\operatorname{cg} 24798010$ & CRB3 & 7717 & $6.26 \mathrm{E}-36$ \\
$\operatorname{cg} 24007193$ & DENND1C & 42180 & $4.17 \mathrm{E}-29$ \\
\hline
\end{tabular}

The 'adj.pval' represents adjusted $P$-value

correlation between every methylation probe and CRB3 mRNA expression in ccRCC. The highest correlation was between CRB3 mRNA expression and probe cg24798010 (Table 3), which resides in the CRB3 gene region without CpG islands (Table 4). The target of probe cg24798010 is shown in Table 4. Using the UCSC website (http:// genome.ucsc.edu/), we found that $\operatorname{cg} 24798010$ resides in the transcription factor EZH2 binding domain (chr19: 6464129-6465140).

\section{Association of cg24798010 methylation and pathological features in cCRCC}

We analyzed the association of $\operatorname{cg} 24798010$ methylation and pathological features of ccRCC using the TCGA data (Table S1). The results showed that cg24798010 methylation
Table 2 Correlation of CRB3 probe DNA methylation with CRB3 mRNA expression

\begin{tabular}{ll}
\hline Probe & $P$ value \\
\hline $\operatorname{cg} 03258472$ & $8.8817841970013 \mathrm{E}-16$ \\
$\operatorname{cg} 11715228$ & $2.2204460492503 \mathrm{E}-16$ \\
$\operatorname{cg} 05384271$ & $-2.2204460492503 \mathrm{E}-16$ \\
$\operatorname{cg} 14782015$ & $-6.6613381477509 \mathrm{E}-16$ \\
$\operatorname{cg} 25637722$ & 0 \\
$\operatorname{cg} 17826168$ & $1.1102230246252 \mathrm{E}-16$ \\
$\operatorname{cg} 27072749$ & $-2.2204460492503 \mathrm{E}-16$ \\
$\operatorname{cg} 26644059$ & $2.2204460492503 \mathrm{E}-16$ \\
$\operatorname{cg} 17799295$ & $6.6613381477509 \mathrm{E}-16$ \\
$\operatorname{cg} 09761352$ & $4.4408920985006 \mathrm{E}-16$ \\
$\operatorname{cg} 01216308$ & 0 \\
$\operatorname{cg} 24798010$ & 0 \\
$\operatorname{cg} 24007193$ & $3.3306690738755 \mathrm{E}-16$ \\
\hline
\end{tabular}

$P$ values were generated using Linear Regression

correlated with laterality, pathologic stage, $\mathrm{T}$ stage, $\mathrm{M}$ stage, and neoplasm-histologic-grade, but did not correlate with $\mathrm{N}$ stage, race, or ethnicity (Fig. 5).

\section{Restoration of CRB3 mRNA by DNA methylation inhibitor decitabine in ccRCC cell lines}

To validate the association of methylation and CRB3 mRNA levels, we tested the effect of Decitabine, a methyltransferase inhibitor, on the expression of CRB3. The test showed that the CRB3 mRNA level was higher in the cells treated with Decitabine than the control cells (Fig. 6a, b). With the concentration of Decitabine increased, the mRNA levels of CRB3 increased (Fig. 6a, b). These results support that there is a relationship between the decreased expression of CRB3 mRNA and high methylation of CRB3 in ccRCC. 
Table 3 Correlation of CRB3 DNA methylation with CRB3 mRNA expression

\begin{tabular}{lccl}
\hline Probe & \multicolumn{2}{l}{ Spearman coefficient (Meth vs } & Correlation \\
& mRNA) & & \\
\hline & Normal & Tumor & \\
$\operatorname{cg} 03258472$ & -0.337 & 0.037 & \\
$\operatorname{cg} 11715228$ & -0.383 & -0.079 & \\
$\operatorname{cg} 05384271$ & -0.381 & 0.023 & \\
$\operatorname{cg} 14782015$ & -0.403 & -0.138 & Weak \\
$\operatorname{cg} 25637722$ & -0.364 & -0.121 & Weak \\
$\operatorname{cg} 17826168$ & 0.017 & -0.165 & Weak \\
$\operatorname{cg} 27072749$ & 0.055 & -0.249 & Weak \\
$\operatorname{cg} 26644059$ & -0.151 & -0.266 & Weak \\
$\operatorname{cg} 17799295$ & 0.118 & -0.318 & Moderate \\
$\operatorname{cg} 09761352$ & -0.076 & -0.089 & Weak \\
$\operatorname{cg} 01216308$ & -0.465 & -0.369 & Moderate \\
$\operatorname{cg} 24798010$ & -0.3 & -0.472 & Moderate \\
$\operatorname{cg} 24007193$ & 0.363 & 0.011 & \\
\hline
\end{tabular}

'Meth' represents methylation

\section{Discussion}

In this study, we found that CRB3 mRNA expression decreased in ccRCC. We also demonstrated that increased CRB3 DNA methylation was negatively correlated with CRB3 mRNA expression. These findings indicate that CRB3 DNA methylation reduces CRB3 mRNA expression in ccRCC, which might be the mechanism underlying the reduction in $\mathrm{CRB} 3$ protein expression in ccRCC [21]. CRB3 protein expression in ccRCC was negatively associated with TNM stage, pathological grade, and poor
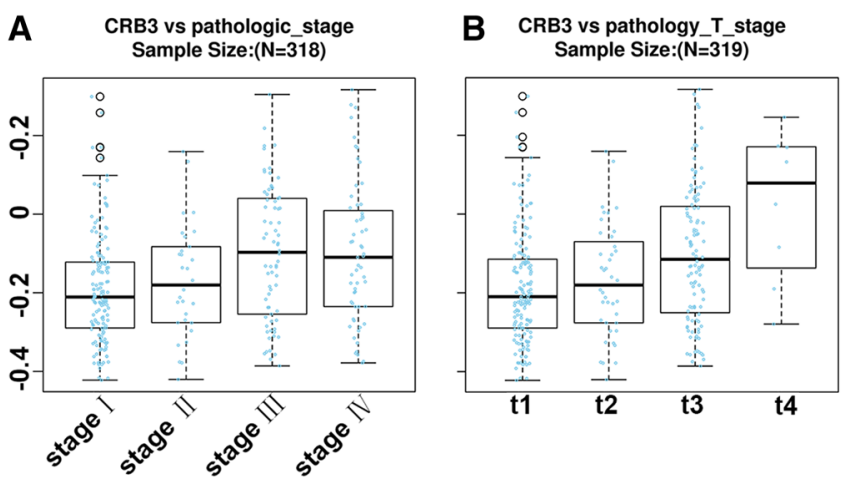

Fig. 3 Association of CRB3 DNA methylation and pathological features in ccRCC. a: CRB3 DNA methylation with pathologic stage $(P=2.134 \mathrm{e}-04, \mathrm{n}=318), \mathbf{b}:$ CRB3 DNA methylation with $\mathrm{T}$ stage $(P=1.004 \mathrm{e}-04, \mathrm{n}=319)$; $\mathbf{c}$ : CRB3 DNA methylation with $\mathrm{N}$ stage $(P=2.383 \mathrm{e}-02, \mathrm{n}=141) ; \mathbf{d}$ : CRB3 DNA methylation with $M$ stage

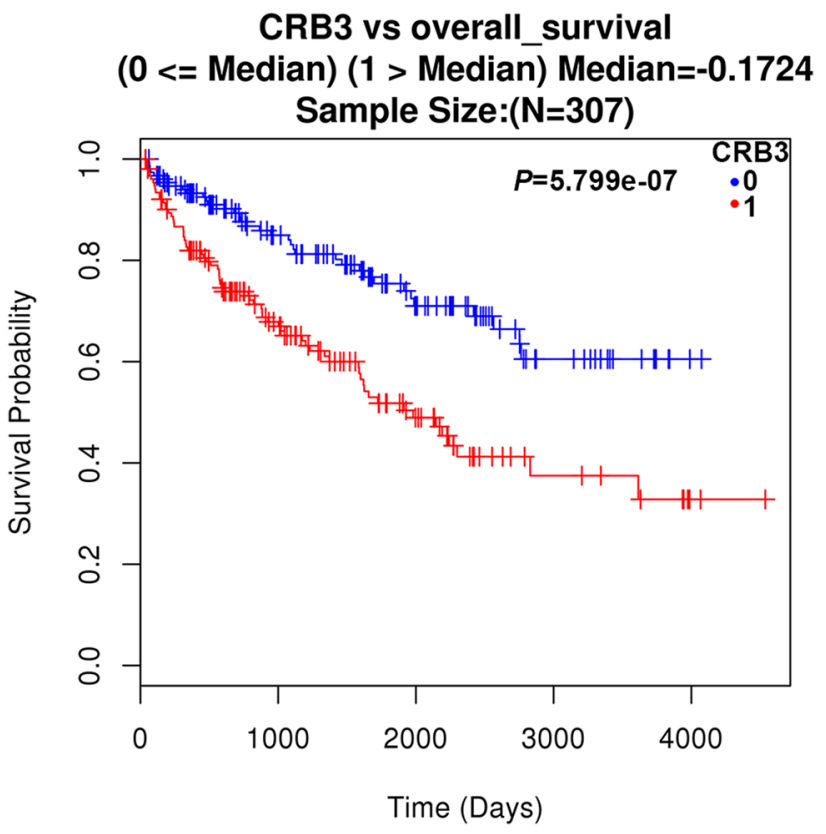

Fig. 4 Correlation of overall survival with CRB3 DNA methylation in ccRCC. Kaplan-Meier plots were produced using LinkedOmics (http://www.linkedomics.org/login.php) $(P=5.799 \mathrm{e}-07) . \quad P$-values were generated by Kaplan-Meier plots

prognosis of ccRCC patients [21]. CRB3 DNA methylation correlated with pathologic stage, $\mathrm{T}$ stage, $\mathrm{N}$ stage, $\mathrm{M}$ stage, and poor prognosis in ccRCC patients. Methylation of the CRB3 probe $\operatorname{cg} 24798010$ correlated with laterality, pathologic stage, $\mathrm{T}$ stage, $\mathrm{M}$ stage, and neoplasm histologic grade, but not with $\mathrm{N}$ stage or race. Collectively, these results suggest that CRB3 DNA methylation decreases CRB3 mRNA and protein expression, promotes ccRCC progression, and reduces overall survival of ccRCC patients.
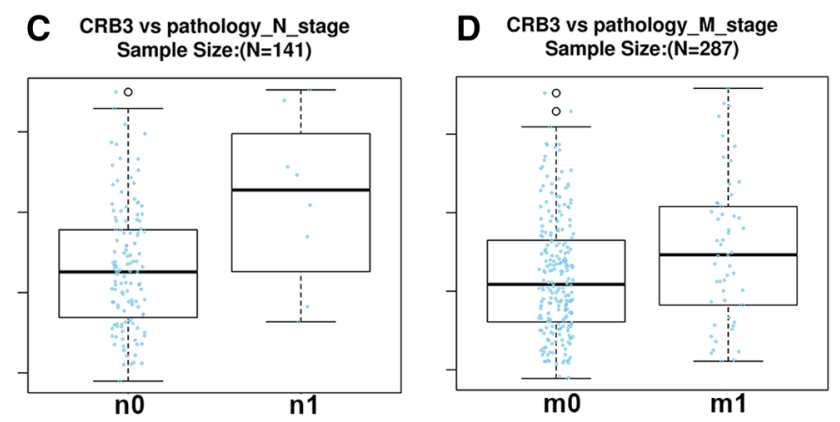

$(P=1.488 \mathrm{e}-02, \mathrm{n}=287)$. Box plots were produced using LinkedOmics (http://www.linkedomics.org/login.php). $\mathbf{a}$ and b Statistically tested using the Kruskal-Wallis test, and $\mathbf{c}$ and $\mathbf{d}$ were tested using the Wilcox test 
Table 4 The specific

information of $\operatorname{cg} 24798010$

\begin{tabular}{llllllll}
\hline Probe & Chr & cg_start & cg_end & probe_start & probe_end & gene_start & gene_end \\
\hline cg24798010 & chr19 & 6464885 & 6464886 & 6464837 & 6464886 & 6463788 & 6467232 \\
\hline
\end{tabular}

A

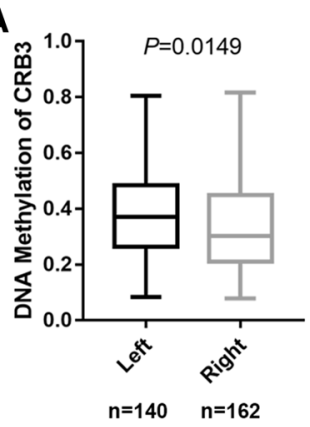

E

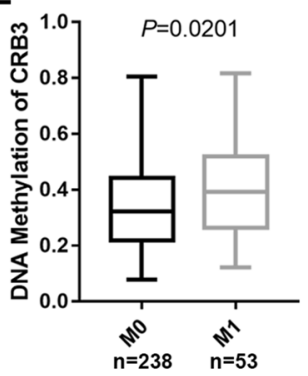

B

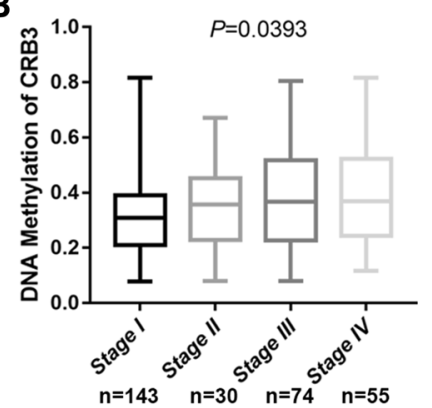

$\mathbf{F}$

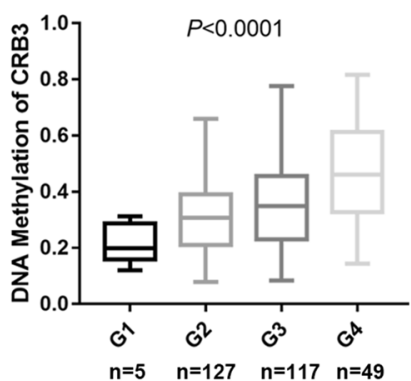

Fig. 5 Association of cg24798010 methylation and pathological features in ccRCC. a, cg24798010 methylation was higher in the left kidney compared to the right kidney $(P=0.0149)$; b, cg24798010 methylation increased as the pathological stage increased from stage I, II, III, to IV $(P=0.0393)$; c, cg24798010 methylation increased as the pathological stage increased from T1, T2, T3, to T4 $(P=0.004)$; e, cg24798010 methylation was higher at M1 compared to M0

A

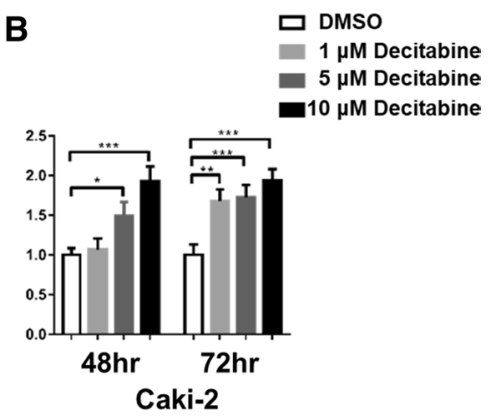

Fig. 6 Restoration of CRB3 mRNA by DNA methylation inhibitor Decitabine in ccRCC cell lines. Cakl-1(a) and Cakl-2(b) cells were incubated with $1,5,10 \mu \mathrm{M}$ Decitabine for $48 \mathrm{~h}$ or $72 \mathrm{~h}$, and the mRNA levels of CRB3 were detected by Real-time PCR. All data was represented as mean \pm SEM $(\mathrm{n}=3, * P<0.05, * * P<0.01$, $* * * P<0.001)$
C

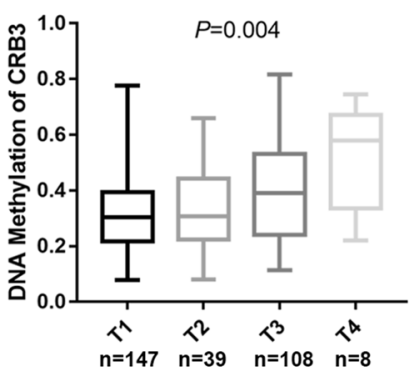

D

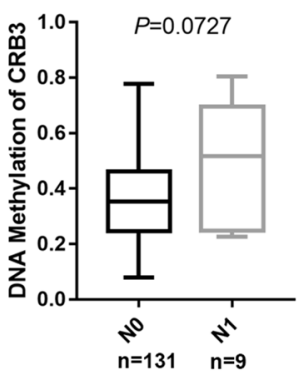

G

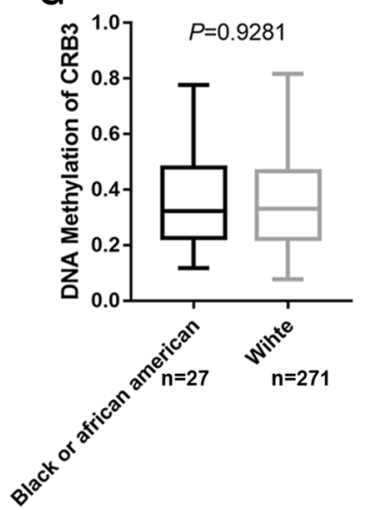

H

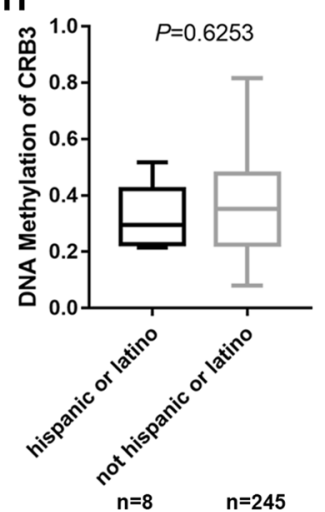

$(P=0.0201) ; \mathbf{f}, \operatorname{cg} 24798010$ methylation increased as the pathological stage increased from stage G1, G2, G3, to G4 $(P<0.001)$. There was no difference in cg24798010 methylation with regards to $\mathrm{N}$ stage and race (d, N0 and N1; g, black and white; H, Hispanic or Latino and non-Hispanic or Latino). $P$-values were generated using the Mann-Whitney test (a, d, e, g and h) and Kruskal-Wallis test (b, c and f)

DNA methylation often represents a potential biomarker for early detection, prognosis, and therapeutic response in ccRCC [3]. Nineteen CpG methylation sites for ccRCC, which were identified using TCGA and gene expression omnibus (GEO), can be used to distinguish between high- and low-risk patients and improve the predictive ability of the tumor node metastasis staging system [28]. Some potential prognostic methylation markers for RCC have been identified, including BNC1, SCUBE3, GATA5, SFRP1, GREM1, RASSF1A, PCDH8, LAD1, and NEFH [3]. Furthermore, Lin et al. found that PCDH17 methylation in serum was a frequent event in RCC and associated with poor outcomes [29].

DNA methylation is an early event in the process of carcinogenesis and gradually increases during tumor progression. Therefore, DNA methylation levels of precancerous lesions or early tumors is one of the most promising 
methods for early diagnosis of cancer. In this study, we found that high CRB3 DNA methylation levels decreased CRB3 mRNA expression, and further increased the malignancy, invasion, and metastasis of renal tumors, as well as decreased the survival time of ccRCC patients. These findings indicate that DNA methylation of CRB3 can be both a prognostic marker and therapeutic target for ccRCC.

Acknowledgements This work was financially supported by the National Natural Science Foundation of China (81672876, 81702631).

\section{Compliance with ethical standards}

Conflict of interest The authors declare no conflicts of interest.

Open Access This article is distributed under the terms of the Creative Commons Attribution 4.0 International License (http://creativecommons.org/licenses/by/4.0/), which permits unrestricted use, distribution, and reproduction in any medium, provided you give appropriate credit to the original author(s) and the source, provide a link to the Creative Commons license, and indicate if changes were made.

\section{References}

1. Siegel RL, Miller KD, Jemal A (2018) Cancer statistics, 2018. CA Cancer J Clin 68:7-30

2. Hsieh JJ, Purdue MP, Signoretti S et al (2017) Renal cell carcinoma. Nat Rev Dis Primers 3:17009

3. Joosten SC, Deckers IA, Aarts MJ et al (2017) Prognostic DNA methylation markers for renal cell carcinoma: a systematic review. Epigenomics 9:1243-1257

4. Posadas EM, Limvorasak S, Figlin RA (2017) Targeted therapies for renal cell carcinoma. Nat Rev Nephrol 13:496-511

5. Zarrabi K, Wu S (2018) Current and emerging therapeutic targets for metastatic renal cell carcinoma. Curr Oncol Rep 20:41

6. Rodriguez-Vida A, Hutson TE, Bellmunt J, Strijbos MH (2017) New treatment options for metastatic renal cell carcinoma. ESMO Open 2:e000185

7. Barata PC, Rini BI (2017) Treatment of renal cell carcinoma: current status and future directions. CA Cancer J Clin 67:507-524

8. Jones PA, Baylin SB (2007) The epigenomics of cancer. Cell 128:683-692

9. Woods Iii LC, Li Y, Ding Y et al (2018) DNA methylation profiles correlated to striped bass sperm fertility. BMC Genom 19:244

10. Baylin SB (2005) DNA methylation and gene silencing in cancer. Nat Rev Clin Oncol 2(S1):S4

11. Morris MR, Maher ER (2010) Epigenetics of renal cell carcinoma: the path towards new diagnostics and therapeutics. Genome Med 2:59

12. Morris MR, Latif $F$ (2016) The epigenetic landscape of renal cancer. Nat Rev Nephrol 13:47

13. Margolis B (2018) The Crumbs3 polarity protein. Cold Spring Harb Perspect Biol 10:a027961
14. Gao Y, Lui WY, Lee WM, Cheng CY (2016) Polarity protein Crumbs homolog-3 (CRB3) regulates ectoplasmic specialization dynamics through its action on F-actin organization in Sertoli cells. Sci Rep 6:28589

15. Szymaniak AD, Mahoney JE, Cardoso WV, Varelas X (2015) Crumbs3-mediated polarity directs airway epithelial cell fate through the hippo pathway effector yap. Dev Cell 34:283-296

16. Yin Y, Sheng J, Hu R, Yang Y, Qing S (2014) The expression and localization of $\mathrm{Crb} 3$ in developmental stages of the mice embryos and in different organs of 1-week-old female mice. Reprod Domest Anim 49:824-830

17. Loie E, Charrier LE, Sollier K, Masson JY, Laprise P (2015) CRB3A controls the morphology and cohesion of cancer cells through Ehm2/p114RhoGEF-dependent signaling. Mol Cell Biol 35:3423-3435

18. Whiteman EL, Fan S, Harder JL et al (2014) Crumbs 3 is essential for proper epithelial development and viability. Mol Cell Biol 34:43-56

19. Charrier LE, Loie E, Laprise P (2015) Mouse Crumbs 3 sustains epithelial tissue morphogenesis in vivo. Sci Rep 5:17699

20. Karp CM, Tan TT, Mathew R et al (2008) Role of the polarity determinant crumbs in suppressing mammalian epithelial tumor progression. Cancer Res 68:4105-4115

21. Mao X, Li P, Ren Y et al (2015) Cell polarity protein CRB3 is an independent favorable prognostic factor for clear cell renal cell carcinoma. Int J Oncol 46:657-666

22. Issa JP (2012) DNA methylation as a clinical marker in oncology. J Clin Oncol 30:2566

23. Jones PA (2012) Functions of DNA methylation: islands, start sites, gene bodies and beyond. Nat Rev Genet 13:484

24. Chandrashekar DS, Bashel B, Sah B et al (2017) UALCAN: a portal for facilitating tumor subgroup gene expression and survival analyses. Neoplasia 19:649-658

25. Díez-Villanueva A, Mallona I, Peinado MA (2015) Wanderer, an interactive viewer to explore DNA methylation and gene expression data in human cancer. Epigenet Chromatin 8(1):22

26. Coleman WB, Kumar D, Hassan MK, Pattnaik N, Mohapatra N, Dixit M (2017) Reduced expression of IQGAP2 and higher expression of IQGAP3 correlates with poor prognosis in cancers. PLoS ONE 12:e0186977

27. Vasaikar SV, Straub P, Wang J, Zhang B (2018) LinkedOmics: analyzing multi-omics data within and across 32 cancer types. Nucleic Acids Res 46:D956-D963

28. Peng D, Ge G, Xu Z et al (2018) Diagnostic and prognostic biomarkers of common urological cancers based on aberrant DNA methylation. Epigenomics 10:1189

29. Lin Y-L, Wang Y-P, Li H-Z, Zhang X (2017) Aberrant promoter methylation of PCDH17 (protocadherin 17) in serum and its clinical significance in renal cell carcinoma. Med Sci Monit 23:3318-3323

Publisher's Note Springer Nature remains neutral with regard to jurisdictional claims in published maps and institutional affiliations. 